\title{
O tutor como agente facilitador no processo de ensino e aprendizagem: uma experiência
}

\section{na disciplina de Bioquímica Metabólica} The tutor as facilitator agent in the teaching and learning process: an experience in the discipline of
Metabolic Biochemistry

Lucas Coêlho Bernardoㅁ, Nathália Regina Galvão Silva², Augusto Cézar Vasconcelos de Freitas Júnior ${ }^{3}$, Cícero Francisco Bezerra Felipe ${ }^{3}$, Davi Felipe Farias ${ }^{3, *}$

${ }^{1}$ Programa de Pós-Graduação em Biologia Aplicada à Saúde, Universidade Federal de Pernambuco, Recife, PE, Brasil.

${ }^{2}$ Programa de Pós-Graduação em Ciências Biológicas, Universidade Federal de Pernambuco, Recife, PE, Brasil.

${ }^{3}$ Departamento de Biologia Molecular, Centro de Ciências Exatas e da Natureza, Universidade Federal da

Paraíba, João Pessoa, PB, Brasil.

*davi@dbm.ufpb.br

\begin{abstract}
The tutoring is part of the academic programs offered by the Federal University of Paraiba (UFPB), aiming to help students from the implementation of strategies that facilitate the students' journey through the disciplines with the highest rates of retention and dropout. This work aimed to evaluate the role of the tutor as an agent in the teaching and learning process in the discipline of Metabolic Biochemistry, at UFPB. To that end, the tutors of this discipline applied four directed studies and a didactic game in the classes of 2016.2 (Group 1/n=27) and 2017.1 (Group 2/n= 23) periods. The performance of the students who were benefited from the didactic resources was satisfactory and significantly higher than the group that did not participate. From the evaluation of the tutoring by the students through an online questionnaire, it was unanimous that the discipline of Metabolic Biochemistry must provide tutors, stating that they are important supporters in the teaching and learning process.
\end{abstract}

Keywords: Directed study; didatic game; tutoring.

\section{Resumo}

A tutoria faz parte dos programas acadêmicos oferecidos pela Universidade Federal da Paraíba (UFPB), com objetivo de auxiliar os discentes a partir da implementação de estratégias que facilitem o percurso dos estudantes pelas disciplinas com maiores índices de reprovação e evasão. Este trabalho teve como objetivo avaliar o papel do tutor como agente no processo de ensino e aprendizagem na disciplina de Bioquímica Metabólica, na UFPB. Para tanto, os tutores dessa disciplina aplicaram quatro estudos dirigidos e um jogo didático nas turmas dos períodos letivos 2016.2 (Grupo $1 / n=27$ ) e 2017.1 (Grupo 2/n= 23). O desempenho dos discentes que usufruíram dos recursos didáticos foi satisfatório e significativamente maior do que o grupo que não participou. A partir da avaliação da tutoria pelos discentes por meio de um questionário online, respondeu-se unanimemente que a disciplina de Bioquímica Metabólica precisa ofertar tutores, declarando que os mesmos são facilitadores importantes no processo de ensino e aprendizagem.

Palavras-chave: Estudos dirigidos; Jogo didático; Tutoria. 


\section{Ficha de atividade desenvolvida}

Título $\quad$ O tutor como agente facilitador no processo de ensino e aprendizagem: uma experiência na disciplina de Bioquímica Metabólica

Público alvo Estudantes da graduação

Disciplinas Bioquímica Metabólica

relacionadas

Objetivos Avaliar o papel do tutor como agente no processo de ensino e aprendizagem na educacionais disciplina de Bioquímica Metabólica, na Universidade Federal da Paraíba. Para tanto, foram criadas e adotadas duas abordagens didáticas distintas (desenvolvimento de estudos dirigidos e de um jogo didático), bem como, foi avaliada a importância do tutor de acordo com a percepção dos discentes da disciplina.

Justificativa Uma estratégia que vem sendo utilizada a fim de facilitar o processo de ensino e de uso aprendizagem nas universidades, é por meio da promoção de programas de tutoria. Neste âmbito, a tutoria tem como objetivo auxiliar os discentes a partir da implementação de estratégias alternativas ou complementares que facilitem o percurso dos estudantes pelas disciplinas com maiores índices de reprovação e evasão. Assim, o tutor é responsável por promover plantões de tira-dúvidas, bem como, trazer propostas e incentivar os discentes a utilizarem diferentes recursos didáticos, tais como estudos dirigidos e jogos didáticos.

Conteúdos Bioquímica da digestão e absorção dos nutrientes da dieta; Metabolismo dos trabalhados carboidratos; Ciclo do ácido cítrico; e Cadeia respiratória e fosforilação oxidativa.

Duração 8 meses, distribuído em 4 meses de acompanhamento com uma turma para a estimada aplicação de estudos dirigidos e 4 meses para aplicação de um jogo didático com uma turma diferente.

Materiais Estudos dirigidos, Software Power Point, impressão em papel cochê (tabuleiro usados do jogo confeccionado), peças para o jogo de tabuleiro, como pinos e dados.

Link https://docs.google.com/forms/d/e/ 1FAIpQLSeLRyf8cZ5DSEU7iUzSQahN25R8OATOMHCCzeYxi47TGTDO wg/viewform?usp=sf_link 


\section{Introdução}

A disciplina de Bioquímica trata da compreensão das biomoléculas envolvidas nas vias metabólicas presentes nos sistemas biológicos de organismos vivos, incluindo desde conceitos e funções químicas, até a caracterização dos grupos funcionais presentes em suas estruturas moleculares [1].

No ensino da Bioquímica são percebidas muitas dificuldades em relação ao aprendizado dos estudantes nas instituições de ensino superior. Um fator complicador desse cenário, é que as matrizes curriculares de vários cursos de graduação alocam a disciplina de Bioquímica já nos primeiros anos do curso, atuando como pré-requisito para outros componentes [2].

Dentre os principais obstáculos relatados pelos estudantes de Bioquímica de vários cursos de graduação, destaca-se a dificuldade em compreender os conteúdos ministrados devido à linguagem complexa e conceitos abstratos [3]. Consequentemente, os discentes acabam desenvolvendo aversão à disciplina, o que gera baixo rendimento durante as avaliações. Para que essa situação seja mudada, é essencial que o uso de abordagens centradas no discente, a qual se dedique a solução de problemas e não na prática de coletar informações [4,5,6]. Desta forma, é necessário desenvolver novas ferramentas educacionais a fim de facilitar a compreensão desses conteúdos, como também inserir o discente de maneira ativa na construção do seu conhecimento.

Sabendo que somente as aulas expositivas não são eficazes em proporcionar um processo de ensino e aprendizado adequado, a utilização de metodologias alternativas tem permitido a formação crítica e reflexiva aos estudantes. O docente pode recorrer a recursos didáticos práticos e aplicáveis como questionários ou mapas conceituais, os quais podem aumentar o desempenho acadêmico dos discentes [6].

O estudo dirigido é uma ferramenta didática com objetivo de questionar sobre determinados assuntos, tornando o estudante independente do educador. Assim, o docente é um intermediário entre os questionários e mapas conceituais, que estimulam e incentivam o discente a ser o construtor do seu próprio conhecimento [7].

As metodologias didáticas são utilizadas como uma saída para o ensino tradicional. Essas, por sua vez, permitem ao discente aprender de forma lúdica, como por exemplo, por meio do uso de jogos didáticos, que tendem a criar um ambiente diferenciado e despertam a curiosidade sobre um determinado conteúdo [8].

Outra estratégia que vem sendo utilizada a fim de facilitar o processo de ensino e aprendizado nas universidades, é por meio da promoção de programas de monitoria e 
tutoria. Neste âmbito, a tutoria faz parte dos programas acadêmicos oferecidos pela Universidade Federal da Paraíba, que tem como objetivo auxiliar os discentes a partir da implementação de estratégias alternativas ou complementares que facilitem o percurso dos estudantes em disciplinas com maiores índices de reprovação e evasão. Estudantes com bons rendimentos em determinados componentes curriculares são selecionados e então chamados de tutores, que acompanham os docentes e discentes em disciplinas básicas dos primeiros períodos do curso de graduação. Assim, o tutor é responsável por promover plantões de tira-dúvidas, bem como trazer propostas e incentivar os discentes a utilizarem diferentes recursos didáticos [9].

Desta maneira, este trabalho teve como objetivo avaliar o papel do tutor como agente no processo de ensino e aprendizagem na disciplina de Bioquímica Metabólica, na Universidade Federal da Paraíba.

\section{Metodologia}

Para atingir o objetivo do estudo foram escolhidas duas turmas, ambas do curso de Ciências Biológicas que cursaram a disciplina de Bioquímica Metabólica durante o período letivo 2016.2 (Grupo 1/n= 27) e 2017.1 (Grupo 2/n= 23), da Universidade Federal da Paraíba (UFPB), Campus I, João Pessoa, Paraíba.

Cada grupo foi acompanhado por um tutor presencial, com disponibilidade de $12 \mathrm{~h} /$ semana e que foi selecionado segundo as normas de um edital próprio da UFPB. Dentre essas normas, está aquela que afirma que os tutores devem ser estudantes que tenham tido desempenho satisfatório no componente curricular específico, tendo conseguido média final $\geq 7,0$. No primeiro contato com as turmas, o tutor disponibilizou horários semanais de plantões tira-dúvidas. Os horários dos encontros foram acordados com ambos os grupos. Além disso, foi criado um grupo para comunicação virtual no aplicativo gratuito para celular Whatsapp.

Como estratégias de fomentar o ensino de Bioquímica, foram desenvolvidos e aplicados estudos dirigidos e um jogo didático, os quais abordaram os seguintes conteúdos programáticos: Unidade I: Bioquímica da digestão e absorção dos nutrientes da dieta; Unidade II: Metabolismo dos carboidratos; Unidade III: Ciclo do ácido cítrico; e Unidade IV: Cadeia respiratória e fosforilação oxidativa (Tabela 1).

Os estudos dirigidos foram aplicados somente com o grupo 1 (27 estudantes de Bioquímica Metabólica do período 2016.2), enquanto o jogo didático destinou-se somente ao grupo 2 (23 estudantes de Bioquímica Metabólica do período 2017.1). 
O tutor como agente facilitador no processo de ensino e aprendizagem: uma experiência na disciplina de Bioquímica Metabólica

Tabela 1. Conteúdo programático dos estudos dirigidos aplicados ao grupo 1 (estudantes do período letivo 2016.2) durante a disciplina de Bioquímica Metabólica.

\begin{tabular}{|c|c|}
\hline \multirow{8}{*}{ UNIDADE I } & Considerações gerais e importância biomédica \\
\hline & Necessidades nutricionais e energéticas em humanos \\
\hline & Os principais macronutrientes da dieta \\
\hline & Digestão e absorção dos macronutrientes \\
\hline & Conceitos e fases do metabolismo \\
\hline & Mapas e vias metabólicas \\
\hline & O papel das vitaminas e minerais \\
\hline & Termodinâmica e regulação \\
\hline \multirow{7}{*}{ UNIDADE II } & Considerações gerais e importância biomédica \\
\hline & A via glicolítica \\
\hline & Fermentação lática e alcoólica \\
\hline & Metabolismo de outras hexoses \\
\hline & Via das pentoses fosfato \\
\hline & Metabolismo do glicogênio e gliconeogênese \\
\hline & Doenças relacionadas a problemas no metabolismo dos carboidratos \\
\hline \multirow{7}{*}{ UNIDADE III } & Considerações gerais e importância bioquímica \\
\hline & Localização e conexão com outras vias metabólicas \\
\hline & Produção de acetil-CoA \\
\hline & Etapas do ciclo \\
\hline & $\begin{array}{l}\text { Produtos formados, rendimento energético e formas de regulação do } \\
\text { ciclo }\end{array}$ \\
\hline & Natureza anfibólica do ciclo do ácido cítrico \\
\hline & Ciclo do glioxilato \\
\hline \multirow{7}{*}{ UNIDADE IV } & Considerações gerais e importância biológica \\
\hline & Localização celular e conexão com outras vias metabólicas \\
\hline & Componentes \\
\hline & Reações de transferência de elétrons em mitocôndrias \\
\hline & Síntese de ATP \\
\hline & Regulação da fosforilação oxidativa \\
\hline & Origem e outras funções das mitocôndrias \\
\hline
\end{tabular}

\subsection{Estudo Dirigido}

Foram aplicados quatro estudos dirigidos (EDs) para o grupo 1 como uma proposta de recurso didático a ser utilizado ao longo do semestre.

O tutor foi o mediador durante o processo de aprendizagem, auxiliando os discentes quando necessário no esclarecimento de dúvidas e na construção das respostas dos EDs. Os EDs respondidos foram entregues ao tutor que foi responsável pela correção dos mesmos, atribuição de notas ( 0 a 10) e retirada de dúvidas sobre o 
conteúdo abordado. De acordo com as unidades de conteúdo da disciplina de Bioquímica Metabólica, os EDs foram estruturados de questões subjetivas, expondo esquemas das vias metabólicas e confrontando situações do cotidiano. As questões dos EDs tinham dificuldade equivalentes àquelas das avaliações da disciplina. Para a correção dos EDs, o tutor foi instruído pelo docente responsável pela disciplina a como conduzir a correção, além de ter fornecido um espelho de correção para auxiliar na avaliação das respostas. Por fim, a correção foi conferida pelo docente responsável.

Posteriormente, foi analisado o desempenho dos estudantes na disciplina a partir das notas finais obtidas por aqueles que compuseram o grupo 1, 27 estudantes que cursaram a disciplina no período letivo de 2016.2. Para tanto, foram comparadas as notas dos estudantes que não fizeram nenhum dos EDs que formaram o subgrupo $1.1(n=10)$ com aqueles que fizeram os EDs e formaram o subgrupo $1.2(n=17)$. A adesão dos discentes à atividade dos EDs foi espontânea e, portanto, resultou em números distintos de participantes entre os subgrupos.

\section{2 Jogo Didático}

Para o grupo 2, estudantes que cursaram a disciplina no período letivo de 2017.1, foi desenvolvido e aplicado um jogo de tabuleiro como recurso didático. $O$ jogo foi composto por um tabuleiro construído utilizando o software PowerPoint 2013 (Microsoft Corporation), impresso em papel couchê, tamanho $A 3$, e contendo dois dados e vários pinos (Figura 1). Durante o uso do jogo, os alunos foram organizados em duplas representadas por pinos que tinham que percorrer o número de casas do tabuleiro correspondente ao número obtido após arremesso dos dados. Quando o pino estacionava numa casa de cor amarela com desenho de uma lupa (Figura 1), o tutor fazia uma pergunta a qual abordava conteúdos vistos em aulas expositivas. Quanto à escolha do quesito a ser perguntado, solicitou-se ao discente a escolha de uma letra de $A$ até W, não sendo válida a escolha da mesma ao longo da rodada. Cada letra representava uma pergunta diferente e com mesmo grau de dificuldade para tentar evitar o episódio de ter uma pergunta mais fácil para uma determinada dupla e uma mais complexa para outra.

Para a análise do impacto desse recurso utilizado pelo tutor e a efetividade do mesmo em sanar dúvidas que surgiram ao longo das rodadas do jogo, foram analisadas questões da avaliação teórica compatíveis com os assuntos abordados durante o jogo didático e comparado o desempenho entre discentes que compareceram (grupo $2.1 / n=7$ ) e que não compareceram (grupo $2.2 / n=16$ ) as tutorias. 
Os estudantes do grupo $2.1(n=7)$ responderam um questionário para avaliar o uso do jogo como uma ferramenta facilitadora e/ou incentivadora no ensino da Bioquímica Metabólica.

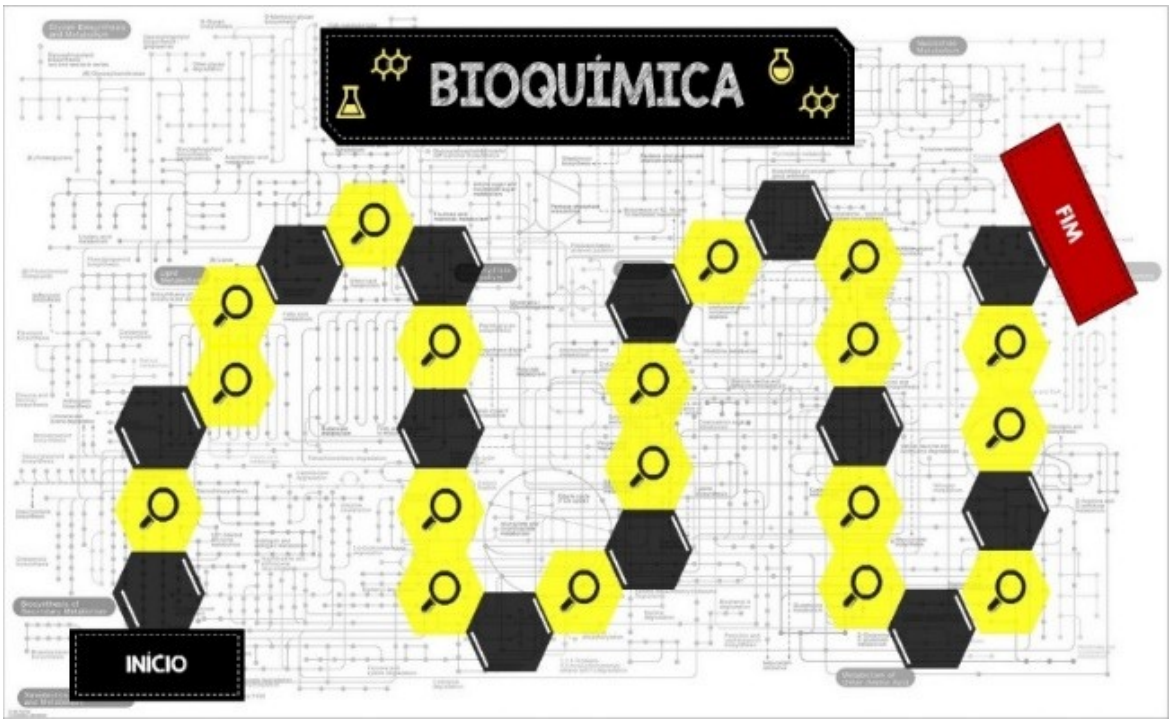

Figura 1. Tabuleiro do jogo didático aplicado ao grupo 2 (discentes do período letivo 2017.1) durante a disciplina de Bioquímica Metabólica.

\subsection{Avaliação da tutoria na disciplina de Bioquímica Metabólica}

Após o término das atividades acadêmicas, criou-se um questionário para avaliação discente das atividades de tutoria desenvolvidas na disciplina de Bioquímica Metabólica (Tabela 2). O questionário foi criado no Google formulários e disponibilizado para os discentes de ambos os grupos no seguinte endereço eletrônico: https://docs.google.com/forms/d/e/1FAlpQLSeLRyf8cZ5DSEU7iUzSQahN25R8OATOMH CCzeYxi47TGTDOwg/viewform?usp=sf link. Os estudantes foram convidados voluntariamente a preencher o questionário, sem qualquer tipo de identificação.

\subsection{Análise de dados}

Os dados foram apresentados como média \pm erro padrão da média. Foram feitas comparações simples ente as médias por meio do teste $t$-Student para amostras independentes, entre as notas dos discentes que participaram das atividades da tutoria e aqueles que não participaram. Essa análise foi feita com o programa GraphPad Prism 6.0 (GraphPad Software Inc., San Diego CA, USA). A significância foi estabelecida com $p<$ 0,05 (intervalo de confiança de 95\%). 
Tabela 2. Questionário estruturado de cunho avaliativo da tutoria de Bioquímica pelo grupo 1 (discentes que cursaram no período de 2016.2) e do grupo 2 (discentes que cursaram em 2017.1).

PERGUNTA

\begin{abstract}
Considera importante a disciplina de Bioquímica Metabólica conter tutores? Por quê?
\end{abstract}

\section{CATEGORIA}

\section{Subjetiva}

\section{Subjetiva}

\begin{tabular}{ll}
\hline $\begin{array}{l}\text { Considera que os tutores atingiram o esperado em tirar suas } \\
\text { dúvidas? }\end{array}$ & ( ) Sim, estou satisfeita(o); \\
( ) Não, acredito que precisam \\
melhorar.
\end{tabular}

Quais horários considera adequado e/ou apropriados para o(a) tutor(a)?
( ) Hora do almoço;
( ) Fim de tarde;
( ) Noite;
( ) De acordo com a turma.

\section{Resultados e Discussão}

Durante a aplicação da metodologia lúdica ao grupo 1, dos 27 discentes apenas 17 destes se dispuseram a realizar os EDs. A avaliação do desempenho nos EDs mostrou que as médias nos ED 1, ED 2, ED 3 e ED 4 foram de $9.38 \pm 0.16,9.5 \pm 0.17,8.31 \pm 0.3$ e $9.38 \pm 0,13$ respectivamente (Figura 2 ).

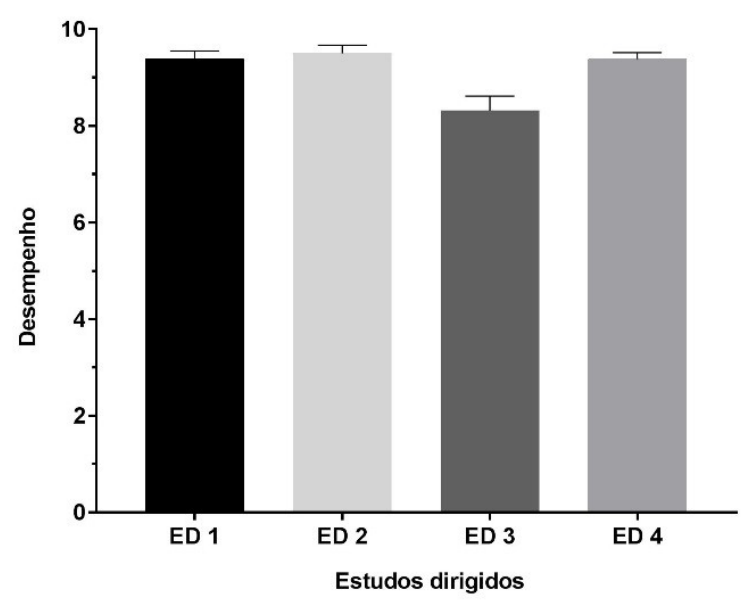

Figura 2. Desempenho (nota) nos estudos dirigidos (ED) do grupo 1 (discentes que cursaram a disciplina em 2016.2). ED 1 - Bioquímica da digestão e absorção dos nutrientes da dieta; ED 2 - Metabolismo dos carboidratos; ED 3 - Ciclo do ácido cítrico; e ED 4 - Cadeia respiratória e fosforilação oxidativa.

O desempenho obtido em todos os EDs foi considerado satisfatório para esse estudo, pois suas médias foram acima da nota 7 a qual é considerada base para aprovação com êxito na disciplina. Interessantemente, os EDs 1, 2 e 4 obtiveram médias superiores a nota 9, demonstrando excelente desempenho dos discentes nos assuntos de digestão e absorção dos nutrientes, metabolismo dos carboidratos e cadeia respiratória. 
A utilização de estratégias metodológicas, como EDs, contribui no desenvolvimento do discente em sala de aula, a partir da exposição da problemática e aprendizado de novos conceitos e vocabulários [10].

Okane e Takahashi [7] relataram que os EDs aumentam a percepção de reconhecimento de dificuldades individuais, respeitando o desenvolvimento particular de estudo, bem como amplia as fontes de busca científica e torna o aluno sujeito ativo de seu aprendizado.

Destaca-se que o plantão de tira-dúvidas da tutoria esteve presente com suas atividades regulares durante os períodos de atividades acadêmicas e com disponibilidade frequente pelo ambiente virtual, a partir do aplicativo gratuito para celular. Segundo Ferreira, Lima e Hornink [11], os aplicativos online proporcionam maior interação entre os estudantes e os demais envolvidos, sendo uma ferramenta eficaz na comunicação de forma imediata.

Ao término do período letivo, foi analisado o desempenho obtido entre discentes pertencentes ao grupo 1, que não fizeram nenhum dos EDs (subgrupo $1.1 / \mathrm{n}=10$ ) e aqueles que fizeram os EDs (subgrupo $1.2 / n=17$ ). Viu-se que o subgrupo 1.2 apresentou a média de $6.32 \pm 0.43$, enquanto o subgrupo 1.1 foi de 2,4 $\pm 0,99$ (Figura 3). Portanto, é notória a diferença $(p<0,05)$ entre os grupos, seja pela eficácia dos EDs, como instrumentos de ensino-aprendizado, ou por fatores subjetivos inerentes a cada discente, tais como motivação e afinidade com a disciplina.

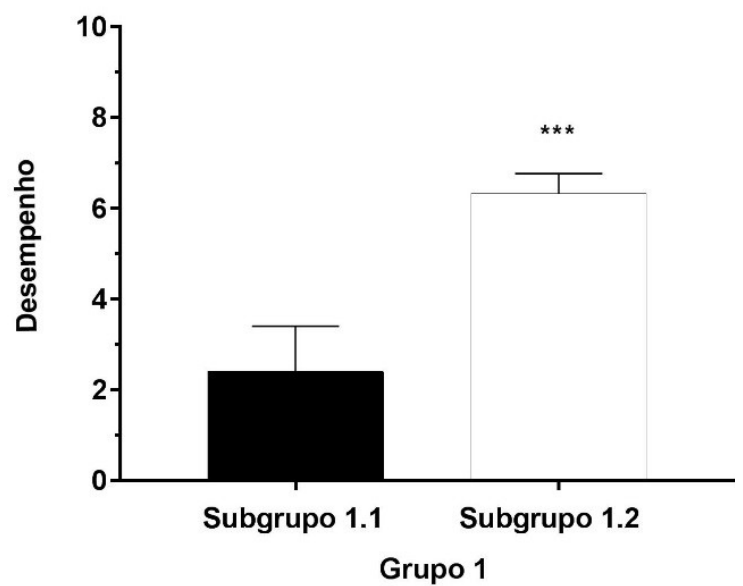

Figura 3. Análise comparativa do desempenho (nota) na disciplina de Bioquímica Metabólica entre os subgrupos 1.1 e 1.2 , obtido ao término do período letivo. ${ }^{* * *} p=0,0004$.

A utilidade dos EDs é observada por Khogali, Laidlaw e Harden [12] ao avaliarem estudantes matriculados na disciplina de Fisiologia Geral, os quais afirmaram que os EDs fazem parte de elementos essenciais para aprimorar o conhecimento de determinado assunto. O que corrobora também com Keyhanmanesh et al. [13], que descreveram efei- 
tos benéficos dos EDs na disciplina de Fisiologia Aplicada, entre estudantes do curso de Odontologia e Medicina, por serem capazes de proporcionar segurança antes das provas avaliativas, promover sensação de desafio e, consequentemente, gradativo aumento no desempenho dos discentes na disciplina.

Vemos que os EDs atuam como um recurso didático de suporte instrucional e são comumente aceitos entre os estudantes [14]. Nesse aspecto, o tutor foi responsável por esquematizar, aplicar e acompanhar o desenvolvimento dos discentes durante cada um dos EDs, a fim de criar um ambiente de desafio e construir um guia de assuntos a serem estudados.

Quanto ao jogo didático, foi utilizado um método comparativo entre os discentes que compareceram a tutoria (subgrupo 2.1) e os que não compareceram (subgrupo 2.2). Para tanto, foram analisadas apenas as questões da avaliação teórica que foram abordadas pelo jogo, que foram um total de 4 questões subjetivas. Os discentes do subgrupo 2.1 apresentaram uma tendência a obter maiores pontuações nessas questões (Figura 3). Entretanto, apenas a questão 4 (Q.4) obteve uma diferença significativa, com $p=0,0068$, o que demonstra que o jogo funcionou bem no entendimento de um aspecto fisiológico do metabolismo dos carboidratos. Ao analisar a média da avaliação teórica da disciplina, o subgrupo 2.1 alcançou $7,84 \pm 1,12(n=7)$ e o subgrupo 2.2 de $3,41 \pm 1,21(n=7)$, essa diferença foi significativa $(p=0,0198)$.

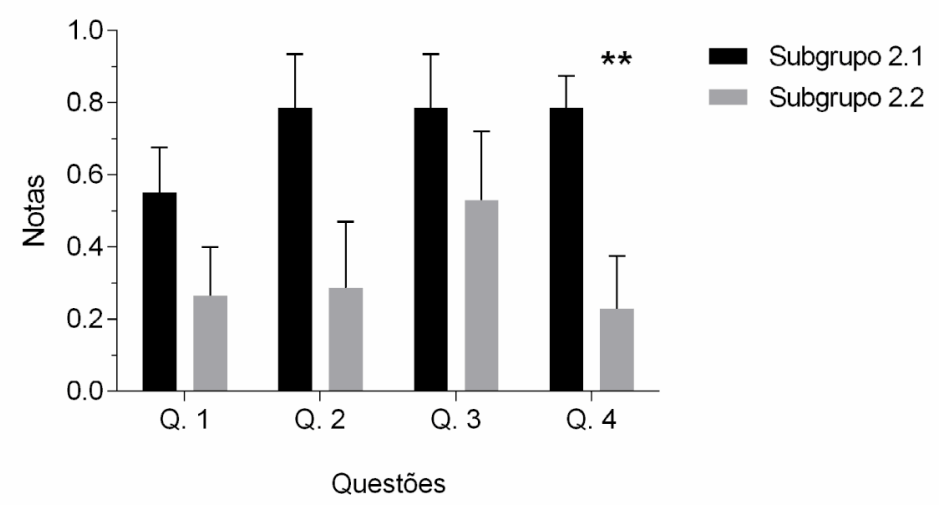

Figura 3. Análise comparativa entre as notas das questões da avaliação teóricas abordadas pelo jogo dos subgrupos 2.1 (os alunos que compareceram as tutorias e utilizaram o jogo) e 2.2 (alunos que não compareceram a tutoria). Q.1 - Descreva sucintamente a digestão do amido (essa questão obteve um $p=$ $0,1460)$. Q.2 - Quais reações reversíveis da via glicolítica são aproveitadas na gliconeogênese? ( $p=$ 0,0565). Q.3 - Escreva as reações que funcionam como pontos de regulação da via glicolítica e informe as enzimas reguladas. $(p=0,3092)$ Q.4 - Qual o efeito no metabolismo dos carboidratos, do glucagon, da insulina e da adrenalina no músculo esquelético? $(p=0,0198) .{ }^{* *} p<0,05$.

Assim, os alunos que obtiveram maior desempenho de notas na avaliação teórica, tiveram maior compreensão do conteúdo de glicólise e gliconeogênese devido ao auxílio 
promovido pelos tutores na aplicação de metodologias alternativas, desta forma, é evidente o potencial do tutor e do uso de ferramentas lúdicas no ensino de Bioquímica.

Nossos resultados mostraram que os discentes que participaram do jogo didático obtiveram um melhor desenvolvimento na disciplina de Bioquímica Metabólica, quando comparados aos que não participaram.

De acordo com Ferreira e Gonçalvez [2], em algumas ocasiões, são executados recursos de fácil aplicabilidade no Ensino Superior que demonstram potencial como mediadores para a consolidação dos conhecimentos dos discentes, porém ainda existem dificuldades de aprimorar esses materiais didáticos e o problema enfrentado no presente estudo pôde ser superado com a aplicabilidade do jogo desenvolvido pelos tutores durante a aula do docente titular, incluindo a participação do mesmo e aumentando a taxa de estudantes com acesso a uma metodologia lúdica e não tradicional.

A utilização da metodologia de jogo didático foi avaliada pelos discentes através de questionários. O recurso utilizado foi avaliado como excelente $(28,6 \%)$ e bom $(71,4 \%)$. E 85,5\% afirmaram que o jogo contribuiu na redução das dúvidas que foram levadas aos plantões da tutoria. Todos os participantes concordaram que a utilização desse método alternativo ao ensino tradicional seria aplicável à disciplina de Bioquímica Metabólica. Além disso, a maioria deles (85\%) relata que, em outras disciplinas, durante a tutoria ou monitoria, foram utilizados jogos didáticos. Isso demonstra que é uma metodologia bastante empregada para auxiliar no processo de ensino e aprendizagem. Após jogar, os discentes relataram a sensação de satisfação e curiosidade (Figura 4).

A

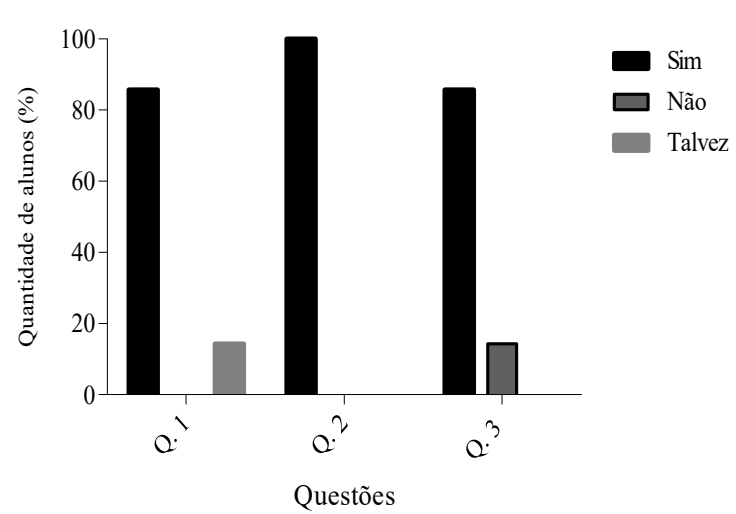

B

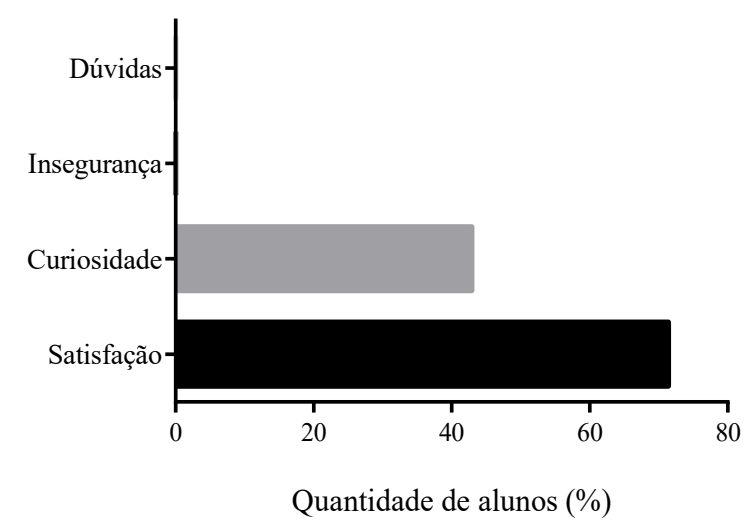

Figura 4. (A) Representação dos resultados das questões objetivas do questionário de avaliação do jogo. Questão 1 (Q.1 - O jogo didático auxiliou nas dúvidas levadas para a tutoria?), questão 2 (Q.2 - A utilização do jogo didático seria aplicável à disciplina de Bioquímica Metabólica?), questão 3 (Q.3 - Outras disciplinas, tutorias ou monitorias que você frequentou utilizaram a metodologia de jogos didáticos?). (B) Resultado da questão referente à sensação após o jogo didático, em relação ao conhecimento. 
Os jogos são considerados educativos quando existe equilíbrio entre o lúdico e o educativo, proporcionando diversão e prazer ao mesmo tempo. Além de consolidar conceitos corretos, introduzir outras definições e compreensão do conhecimento científico [15]. Então, é visível que essa metodologia utilizada na tutoria alcançou o equilíbrio e de fato é educativa, com isso os discentes despertaram interesse pela Bioquímica.

A partir da avaliação da tutoria pelos discentes na plataforma online, respondeuse unanimemente que a disciplina de Bioquímica Metabólica precisa ofertar tutores, declarando que o mesmo é um facilitador no processo de ensino e aprendizagem. Um dos papeis dos tutores nas disciplinas é facilitar a comunicação entre os discentes e o docente e ampliar esse momento de intercâmbio no aprendizado, pois muitas vezes o professor da disciplina tem dificuldade em disponibilizar mais tempo para atendimento a turma. Desta forma, o tutor pode complementar essa carga horária e auxiliar tanto o docente como o discente. Destacando que $85 \%$ dos discentes declararam que o programa de tutoria é importante devido a sua capacidade de promover um incentivo ao desenvolvimento acadêmico dos mesmos. Quanto ao desempenho do tutor, $85 \%$ dos discentes também declararam que o mesmo atingiu as suas expectativas devido a sua habilidade de sanar dúvidas. Quando os discentes foram questionados sobre os horários mais adequados para o atendimento nos plantões de tira-dúvidas, todos afirmaram $(100 \%)$ que preferencialmente deve ser acordado um horário que seja conveniente para a turma. Esse resultado fomenta a importância dos tutores para a disciplina, mas é imprescindível que os mesmos se adéquem aos horários disponíveis da turma para o melhor rendimento desse programa.

O tutor atua como profissional multifacetado e deve guiar no processo ensino e aprendizagem centrado no aluno [16]. No Ensino à Distância, os tutores possuem graus de formação avançados, como mestres e/ doutores, atuando como mediadores do conhecimento prévio aos estudantes por plataformas online ou semipresenciais [17]. Quanto ao Ensino Superior presencial, cabe ao tutor proporcionar apoio em horários distintos daqueles das aulas regulares, oferecer atividades extracurriculares, instruir o discente para uma visão interdisciplinar, como também, compartilhar experiências didático-pedagógicas e intermediar uma troca de saberes acadêmicos [18].

Os programas acadêmicos de apoio às disciplinas propõem contribuir na construção do perfil tanto do tutor quanto do discente, auxiliando o docente da disciplina a partir de interdisciplinaridade e didática dos conteúdos. O que pode ser visto é um resultado da soma entre o tutor e suas abordagens metodológicos facilitadoras de ensino, 
levando ao melhor desenvolvimento e desempenho acadêmico dos envolvidos no processo de ensino e aprendizagem.

A atuação do tutor tende a impactar no ensino de forma significativa, atendendo os discentes individualmente e/ou em grupo. Destacamos que o diferencial da tutoria é dado quanto à flexibilidade nos horários de atendimento do tutor com a turma, permitindo que os mesmos optem pelo horário mais adequado a sua grade, contribuindo para turmas com heterogeneidade de discentes pertencentes a períodos distintos.

Acredita-se que o presente trabalho alcançou o objetivo proposto em descrever o tutor no processo de ensino e aprendizagem, bem como, possibilitou novos víeis para demais docentes e instituições adotarem o programa de tutoria como projeto obrigatório de alto potencial de ensino e aplicação das metodologias alternativas.

\section{Considerações Finais}

O tutor atingiu o objetivo de atuar como agente facilitador no processo de ensino e aprendizagem na disciplina de Bioquímica Metabólica. Mais especificamente, o tutor auxiliou na resolução de estudos dirigidos, ofertou horários compatíveis com a disponibilidade da turma, atingiu o nível esperado na clareza de soluções às dúvidas sobre o conteúdo visto em aulas teóricas e desenvolveu a prática da ludicidade mediante um jogo divertido que envolveu os discentes em uma disciplina com vários assuntos de difícil compreensão.

\section{REFERÊNCIAS}

[1] Barbosa PSB, Gadelha TS, Gadelha CAA, Persuhn DC. Perfil - Biomoléculas. Revista de Ensino de Bioquímica. 2014; 12(1): 24-33.

[2] Ferreira CRC, Gonçalves HJL. Mapeando tendências da pesquisa na área de Educação em Bioquímica da SBBq de 2006 a 2016. Revista de Ensino de Bioquímica. 2017; 15(1): 14-23.

[3] Ramasamy R, Gopal N, Srinivasan A R, Murugaiyan SB. Planning an objective and need based curriculum: the logistics with reference to the undergraduate medical education in biochemistry. Journal of Clinical and Diagnostic Research . 2013; 7(3): 589-594.

[4] Puri D. An integrated problem-based curriculum for biochemistry teaching in medical sciences. Indian Journal of Clinical Biochemistry. 2002; 17(2): 52-59.

[5] Elahi F, Siddiqui MG, Jafarey NA. A retrospective look at the medical curriculum. Journal of the Pakistan Medical Association. 1985; 35(3): 85-92.

[6] Ribeiro JBP, Teles SM, Montenegro MAP, Moreira JR. Intervenção pedagógica e metodologia ativa: o uso da instrução por colegas na educação profissional. Outras Palavras. 2016; 12(2): 1-16.

[7] Okane ESH, Takahashi RT. O estudo dirigido como estratégia de ensino na educação profissional em 
O tutor como agente facilitador no processo de ensino e aprendizagem: uma experiência na disciplina de Bioquímica Metabólica

enfermagem. Revista da Escola de Enfermagem da USP. 2006; 40(2): 160-169.

[8] Farkuh L, Pereira-Leite C. Bioquima 4x: um jogo didático para rever conceitos de bioquímica. Revista de Ensino de Bioquímica. 2014; 12(2): 37-54.

[9] Brasil. Conselho Superior de Ensino, Pesquisa e Extensão (CONSEPE). Regulamento do Programa de Tutoria de Apoio às Disciplinas Básicas. Disponível em

<http://www.de.ufpb.br/departamento/tutoria/Rsep49_2014.pdf>. [Acesso em set 2018].

[10] Conderman G. Developing study guides for middle school learners. The Clearing House: A Journal of Educational Strategies, Issues and Ideas. 2017; 90(2): 60-64.

[11] Ferreira AO, Lima CA, Hornink GG. O ensino aprendizagem online de Bioquímica e as ferramentas de mediação: um estudo de caso. Revista de Ensino de Bioquímica. 2014; 12(1): 5-23.

[12] Khogali SEO, Laidlaw JM, Harden RM. Study guides: a study of different formats. Medical Teachers. 2006; 28(4): 377-379.

[13] Keyhanmanesh R, Bavil FM, Ghojazadeh M, Ebrahimi H, Mazouchian H, Saadatlou MAE. The beneficial effects of applied physiology study guides on dentistry studentes' learning. Research and Development in Medical Education. 2014. 3(2): 105-107.

[14] Conderman G, Hedin L. Differentiating study guides. Intervention in School and Clinica. 2017; 53(1): 1927.

[15] Cunha MB. Jogos no ensino de química: considerações teóricas para sua utilização em sala de aula. Química Nova na Escola. 2012; 34(2): 92-98.

[16] Bottii SHO, Regoi S. Preceptor, supervisor, tutor e mentor: quais são seus papéis?. Revista Brasileira de Educação Médica. 2008; 32(3): 363-373.

[17] Bento L, Nascimento MS, Grenha V, Macedo MV. A concepção de tutores a distância sobre interatividade e a formação em EAD: um estudo de caso. Revista Brasileira de Aprendizagem Aberta e a Distância. 2017; 16(1): 24-35.

[18] Frison LMB. Tutoria: uma prática de ensino autorregulada utilizado no ensino superior. Revista Reflexão e Ação. 2013; 21: 66-81. 